\title{
Numerical Simulation for the 2-D Heat Equation with Derivative Boundary Conditions
}

\author{
Iman. I. Gorial \\ Department of Mathematics, College of Education for Pure Science / Ibn Al-Haitham ,Baghdad University, Iraq
}

\begin{abstract}
In this paper, analytical numerical simulation of the 2-D heat equation with derivative boundary conditions has been presented. The algorithm for the numerical simulation for this equation is based on modified decomposition method. The numerical method has been applied to solve a practical examples and it's results have been compared with exact solution.
\end{abstract}

Keywords: Modified decomposition method, 2-D Heat equation, Derivative boundary conditions.

\section{Introduction}

There are many new analytical approximate methods to solve initial value problems in the literature. Among these, Adomian's decomposition method [1,2] have been received much attention in recent years in applied mathematics in general, and easily handle a wide a class of linear or nonlinear problems. The Adomian technique is based on a decomposition of a solution of a nonlinear functional equation in a series of functions. Each term of the series is obtained from a polynomial generated by a power series expansion of an analytic function.

The main advantage of the ADM is that it can be applied directly for all types of functional equations, linear or nonlinear. Another important advantage is capable of greatly reducing the size of computation work while still maintaining high accuracy of the numerical solution.

In [3] the author compared the Adomian Decomposition Method (ADM) and the Taylor series method by using some particular examples, and showed that the Adomian's technique produced reliable results with a fewer iterations, whereas the Taylor series method suffered from computational difficulties. But in this paper, we will show that both Adomian decomposition method and Taylor series method are equivalents and therefore their convergence is the same in both.

Different modifications of this method and their applications are given in [4-9]

\section{Applying the Modified Adomian's Decomposition Method of Solution 2-D the Heat Equation}

In this section we want to describe how to use modified decomposition method for solving 2-D the heat equation with derivative boundary conditions:

$$
\begin{aligned}
& D_{t} \psi(x, y, t)=D_{x x} \psi(x, y, t)+D_{y y} \psi(x, y, t)+\zeta(x, y, t) \\
& \psi(x, y, 0)=f(x, y), 0 \leq x \leq 1,0 \leq x \leq 1 \\
& \psi_{x}(0, y, t)=g_{1}(y, t), 0<t \leq T \\
& \psi_{y}(x, 0, t)=g_{2}(x, t), 0<t \leq T \\
& \psi_{x}(1, y, t)=g_{3}(y, t), 0<t \leq T \\
& \psi_{y}(x, 1, t)=g_{4}(x, t), 0<t \leq T
\end{aligned}
$$

Where $f, g_{1}, g_{2}, g_{3}, g_{4}$ and $\zeta$ are known functions, $\mathrm{T}$ is given constant.

Now, we use Adomian decomposition method

$$
\psi(x, y, t)=\sum_{n=0}^{\infty} \psi_{n}(x, y, t)
$$

Then equation (1) can be rewritten as

$$
L_{t} \psi(x, y, t)=L_{x x} \psi(x, y, t)+L_{y y} \psi(x, y, t)+\zeta(x, y, t)
$$


Where the differential operators $L_{t}(\cdot)=\frac{\partial}{\partial t}(\cdot), L_{x x}=\frac{\partial^{2}}{\partial x^{2}}$ and $L_{y y}=\frac{\partial^{2}}{\partial y^{2}}$, the applying $L^{-1}$ which is defined as:

$$
L^{-1}=\int_{0}^{t}(\cdot) d t
$$

Then equation (3) become

$$
L^{-1}\left(L_{t} \psi((x, y, t))\right)=L^{-1}\left(L_{x x}(\psi(x, y, t))\right)+L^{-1}\left(L_{y y}(\psi(x, y, t))\right)+L^{-1}(\zeta(x, y, t))
$$

Therefore, we can write,

$$
\psi(x, y, t)=\psi(x, y, 0)+L_{t}^{-1}\left(L_{x x}\left(\sum_{n=0}^{\infty} \psi_{n}\right)\right)+L_{t}^{-1}\left(L_{y y}\left(\sum_{n=0}^{\infty} \psi_{n}\right)\right)+L^{-1}(\zeta(x, y, t))
$$

By Wazwaz [4], the modified decomposition method is based on the assumption that the function $\mathrm{P}(x)$ can be divided into two parts, namely $\mathrm{P}_{1}(x)$ and $\mathrm{P}_{2}(x)$. Under this assumption we set

$$
\mathrm{P}(x)=\mathrm{P}_{1}(x)+\mathrm{P}_{2}(x)
$$

We suggest the following modification

$$
\begin{aligned}
& \Psi_{0}=\mathrm{P}_{1} \\
& \Psi_{1}=\mathrm{P}_{2}+L_{t}^{-1}\left(L_{x x} \Psi_{\mathrm{O}}\right)+L_{t}^{-1}\left(L_{y y} \Psi_{\mathrm{O}}\right) \\
& \Psi_{n+1}=L_{t}^{-1}\left(L_{x x}\left(\sum_{n=0}^{\infty} \Psi_{n}\right)\right)+L_{t}^{-1}\left(L_{y y}\left(\sum_{n=0}^{\infty} \Psi_{n}\right)\right), \mathrm{n} \geq 1
\end{aligned}
$$

\section{Numerical Examples}

Example 1: Consider the problem (1) with the following derivative boundary and initial conditions:

$$
\begin{aligned}
& D_{t} \Psi(x, y, t)=D_{x}^{2} \Psi(x, y, t)+D_{y}^{2} \Psi(x, y, t)-3 e^{x+y-t} \\
& \Psi(x, y, 0)=e^{x+y}, 0 \leq x \leq 1,0 \leq y \leq 1 \\
& \psi_{x}(0, y, t)=e^{y-t}, 0<t \leq T \\
& \psi_{y}(x, 0, t)=e^{x-t}, 0<t \leq T \\
& \psi_{x}(1, y, t)=e^{1+y-t}, 0<t \leq T \\
& \psi_{y}(x, 1, t)=e^{x+1-t}, 0<t \leq T
\end{aligned}
$$

We apply the above modified decomposition method; we obtain

$$
\begin{aligned}
& \Psi_{0}(x, y, t)=e^{x+y-t} \\
& \Psi_{1}(x, y, t)=0 \\
& \Psi_{2}(x, y, t)=0 \\
& \Psi_{3}(x, y, t)=0
\end{aligned}
$$

Then the series form is given by: 


$$
\begin{aligned}
\Psi(x, t) & =\Psi_{0}(x, y, t)+\Psi_{1}(x, y, t)+\Psi_{2}(x, y, t)+\Psi_{3}(x, y, t) \\
= & e^{x+y-t}
\end{aligned}
$$

This is the exact solution $\Psi(x, y, t)=e^{x+y-t}$.

Figure 1 and Figure 2 show the plot of the exact solution surface and the numerical solution surface for 2-D heat equation respectively.

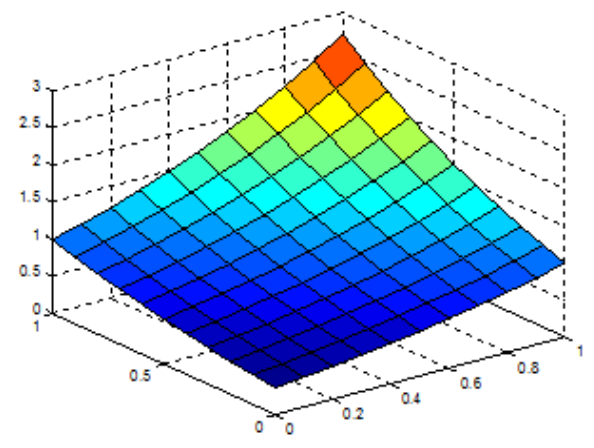

Figure 1: Exact solution

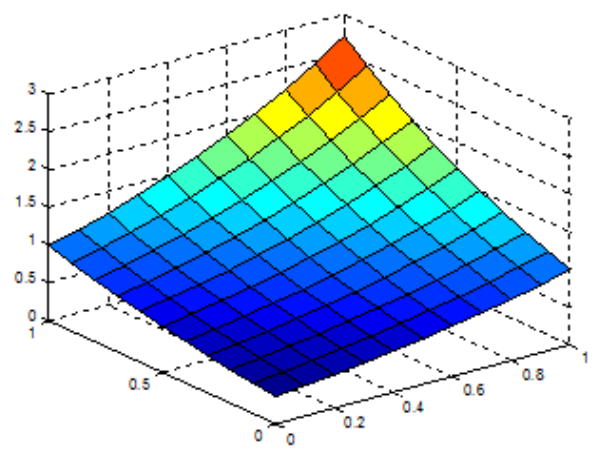

Figure 2: Numerical solution

Example 2: Consider 2-D heat equation with derivative boundary conditions for the equation (1):

$$
\begin{aligned}
& D_{t} \Psi(x, y, t)=D_{x}^{2} \Psi(x, y, t)+D_{y}^{2} \Psi(x, y, t)+e^{-t} \sin (x y) y^{2}+e^{-t} \sin (x y) x^{2}-e^{-t} \sin (x y)+t^{2} x y \\
& \Psi(x, y, 0)=\sin (x y), 0 \leq x \leq 1,0 \leq y \leq 1 \\
& \psi_{x}(0, y, t)=e^{-t} y+\frac{t^{3} y}{3}, 0<t \leq T \\
& \psi_{y}(x, 0, t)=e^{-t} x+\frac{t^{3} x}{3}, 0<t \leq T \\
& \psi_{x}(1, y, t)=e^{-t} \cos (y) y+\frac{t^{3} y}{3}, 0<t \leq T \\
& \psi_{y}(x, 1, t)=e^{-t} \cos (x) x+\frac{t^{3} x}{3}, 0<t \leq T
\end{aligned}
$$

Now we apply the above modified decomposition method, we obtain:

$$
\begin{aligned}
& \Psi_{0}(x, y, t)=e^{-t} \sin (x y)+\frac{1}{3} t^{3} x y \\
& \Psi_{1}(x, y, t)=0 \\
& \Psi_{2}(x, y, t)=0 \\
& \Psi_{3}(x, y, t)=0
\end{aligned}
$$

Then the series form is given by:

$$
\begin{gathered}
\Psi(x, y, t)=\Psi_{0}(x, y, t)+\Psi_{1}(x, y, t)+\Psi_{2}(x, y, t)+\Psi_{3}(x, y, t) \\
=e^{-t} \sin (x y)+\frac{1}{3} t^{3} x y
\end{gathered}
$$

Which gives the exact solution $\Psi(x, y, t)=e^{-t} \sin (x y)+\frac{1}{3} t^{3} x y$.

Figure 3 and Figure 4 show the plot of the exact and the numerical solution surface for 2-D heat equation respectively. 


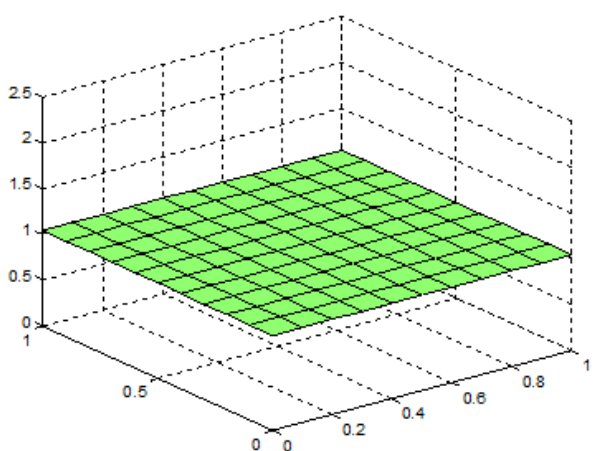

Figure 3: Exact solution

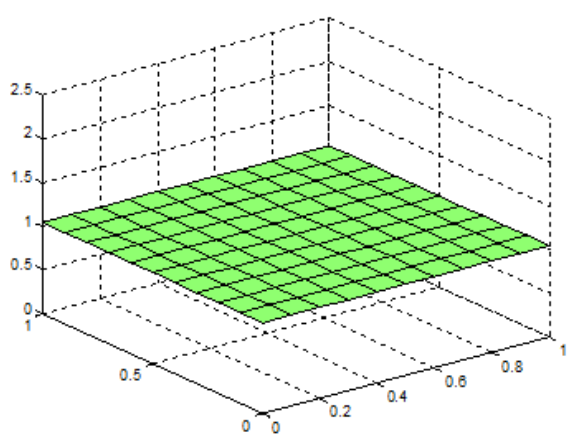

Figure 4: Numerical solution

Example 3: Consider the following:

$$
\begin{aligned}
& D_{t} \Psi(x, y, t)=D_{x}^{2} \Psi(x, y, t)+D_{y}^{2} \Psi(x, y, t) \\
& \Psi(x, y, 0)=e^{2 y} \sin (x), 0 \leq x \leq 1,0 \leq y \leq 1 \\
& \psi_{x}(0, y, t)=e^{2 y+3 t}, 0<t \leq T \\
& \psi_{y}(x, 0, t)=2 e^{3 t} \sin (x), 0<t \leq T \\
& \psi_{x}(1, y, t)=e^{2 y+3 t} \cos (1), 0<t \leq T \\
& \psi_{y}(x, 1, t)=2 e^{2+3 t} \sin (x), 0<t \leq T
\end{aligned}
$$

Now after modified decomposition method, we obtain:

$$
\begin{aligned}
& \Psi_{0}(x, y, t)=e^{2 y+3 t} \sin (x) \\
& \Psi_{1}(x, y, t)=0 \\
& \Psi_{2}(x, y, t)=0 \\
& \Psi_{3}(x, y, t)=0
\end{aligned}
$$

Then the series form is given by:

$$
\begin{gathered}
\Psi(x, y, t)=\Psi_{0}(x, y, t)+\Psi_{1}(x, y, t)+\Psi_{2}(x, y, t)+\Psi_{3}(x, y, t) \\
=e^{2 y+3 t} \sin (x)
\end{gathered}
$$

This is the exact solution $e^{2 y+3 t} \sin (x)$.

The plot of the exact solution surface is shown in Figure 5 and the numerical solution surface is shown in Figure 6 for heat equation

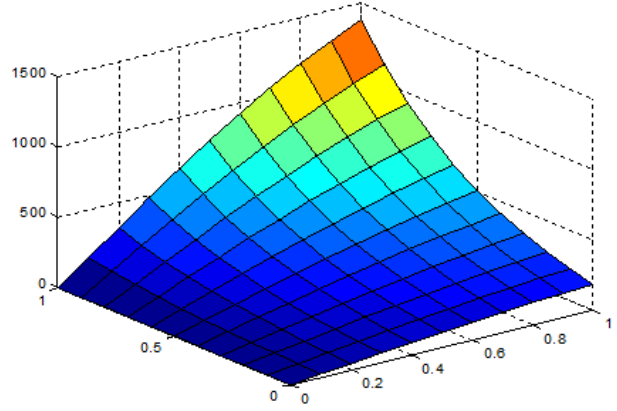

Figure 5: Exact solution

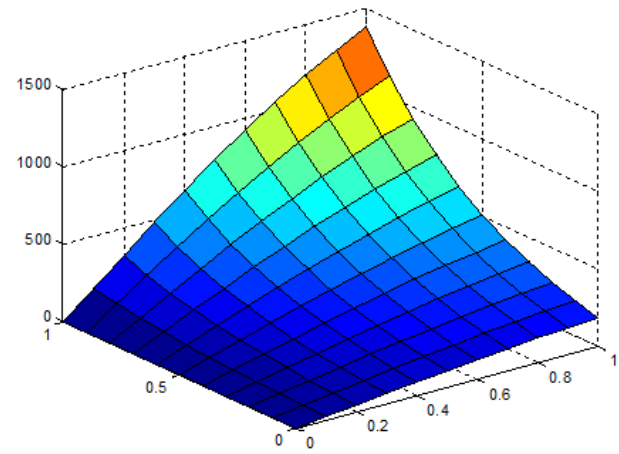

Figure 6: Numerical solution

\section{Conclusion}

In this paper, the results obtained with applied the modified decomposition method of the 2-D heat equation with derivative boundary conditions is accurate. This algorithm is simple and easy to implement. On the other hand, the calculations are easy and take less time than in traditional techniques. 


\section{References}

[1]. Adomian, G. (1986), Nonlinear Stochastic Operator Equations, Academic Press, London.

[2]. Adomian, G. (1994), Solving Frontier Problems of Physics: The Decomposition Method, Kluwer Academic Publishers, Dordrecht.

[3]. A. M. Wazwaz (1998), A comparison between Adomian decomposition method and Taylor series method in the series solution, Appl. Math. Compt. 97, 37-44.

[4]. A. M. Wazwaz, A reliable modification of Adomian decomposition method, Appl. Math. Comput., 102 (1999) 77-86.

[5]. A. M.Wazwaz, A new algorithm for solving differential equations of Lane-Emden type, Appl. Math. Comput., 118 (2001) $287-310$.

[6]. A. M. Wazwaz, A new method for solving singular initial value problems in the second-order ordinary differential equations, Appl. Math. Comput., 128 (2002) 45-57.

[7]. O. Kiymaz, S ,. Mirasyedio glu, A new symbolic computational approach to singular initial value problems in the second-order ordinary differential equations, Appl. Math. Comput., 171 (2005) 1218-1225.

[8]. Iman. I. Gorial (2013), "A Semi-Analytic Method for Solving Two-Dimensional Fractional Dispersion Equation", Advances in Physics Theories and Applications.22: p. 14-18.

[9]. Cheniguel A.( 2011)," Numerical Method for Solving Non-Homogeneous Heat Equation with Derivative Boundary Conditions ", International Mathematical Forum, Vol.6, , no. 14: 651 - 658. 